\title{
Are the Macular and Choroidal Thicknesses Exchange by the Management of Gyrate Atrophy?
}

\author{
Fatih M Adibelli ${ }^{*}$ and Halit Oguz ${ }^{1,2}$ \\ ${ }^{1}$ Department of Ophthalmology, Faculty of Medicine, Harran University, Sanliurfa, Turkey \\ ${ }^{2}$ Department of Ophthalmology, Faculty of Medicine, Medeniyet University, Istanbul, Turkey \\ *Corresponding author: Assist. Prof. Fatih Mehmet ADIBELLI, Department of Ophthalmology, Faculty of Medicine, Harran \\ University, Osmanbey Kampusu, 63100 Sanliurfa, Turkey, Tel: +90-414318-3000, Fax: +90-4143183190
}

\begin{abstract}
Purpose: To identify the macular and choroidal thickness exchange of the case with the gyrate atrophy.

Methods: This is a case report of an 11-year-old boy complaining with deteriorated vision and with multiple peripheral lesions in his fundus in the setting of gyrate atrophy. Spectral domain optical coherence tomography showed, cystoid macular edema was in his both eyes. Plasma ornithine level was found elevated. According to these data, the patients were diagnosed with gyrate atrophy of the choroid and retina. The patient treated with vitamin B6 (pyridoxine) and an arginine-restricted diet.

Results: The macular and choroidal thickness reduced when the patient keep on his diet. After he abandoned the diet, both macular and choroidal thickness became thicker.

Conclusions: This report represents the first case which includes monitoring of the macular and choroidal thickness using spectral-domain optical coherence tomography in a gyrate atrophy case.
\end{abstract}

\section{Keywords}

Gyrate Atrophy, Choroidal thickness, OCT, Ornithine

\section{Introduction}

Gyrate atrophy (GA) of the choroid and retina is a kind of tapetoretinal degeneration which is an autosomal recessive disease characterized by deficiency of ornithine- $\delta$-aminotransferase (OAT). OAT deficiency causes hyperornithinemia, which results in progressive chorioretinal atrophy and increased level of plasma ornithine [1].

Treatment for gyrate atrophy is aimed to the low- ering plasma and urine ornithine levels by arginine restricted diets and vitamin B6 (Pyridoxine) supplement $[2,3]$. Even cystoid macular edema (CME) feature have been described in gyrate edema, it has never been discussed is the macular and choroidal thickness (ChT) altering by the treatment [4].

To the best of our knowledge, this is the first report of GA with the spectral domain optical coherence tomography (SD-OCT) showing alteration during the management for screening choroidal and macular thickening.

\section{Case Report}

An 11-year-old Syrian refugee boy complained of long-term blurred vision in his both eyes for a few years. He was misdiagnosed as Retinitis Pigmentosa in his country. At presentation, best-corrected visual acuity (BCVA) was 20/40 in his right eye (RE), and 20/32 in his left eye (LE). The refractive error was $-5,75$ diopter (D) in the RE and -5.25 D in the LE. Both eyes intraocular pressure was found within normal limits. On his fundus examination detected severe, well-demarcated multiple widespread chorioretinal atrophy involving the mid-periphery bilateral (Figure 1). Cystoid macular edema was evident in his both eyes on SD-OCT. Elevated levels of plasma ornithine at $889 \mu \mathrm{mol} / \mathrm{L}$ (Reference range: $10-163$ ) and urine ornithine at $4206.22 \mu \mathrm{mol} / \mathrm{g}$ Kre (Reference range: 31-91) were also detected. The clinical diagnosis was consistent with GA of the choroid and retina. The patient treated with vitamin B6 (pyridoxine) $300 \mathrm{mg}$ daily and an arginine-restricted diet.

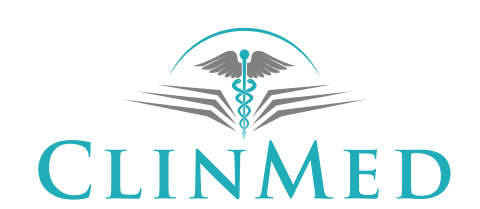

INTERNATIONAL LIBRARY 


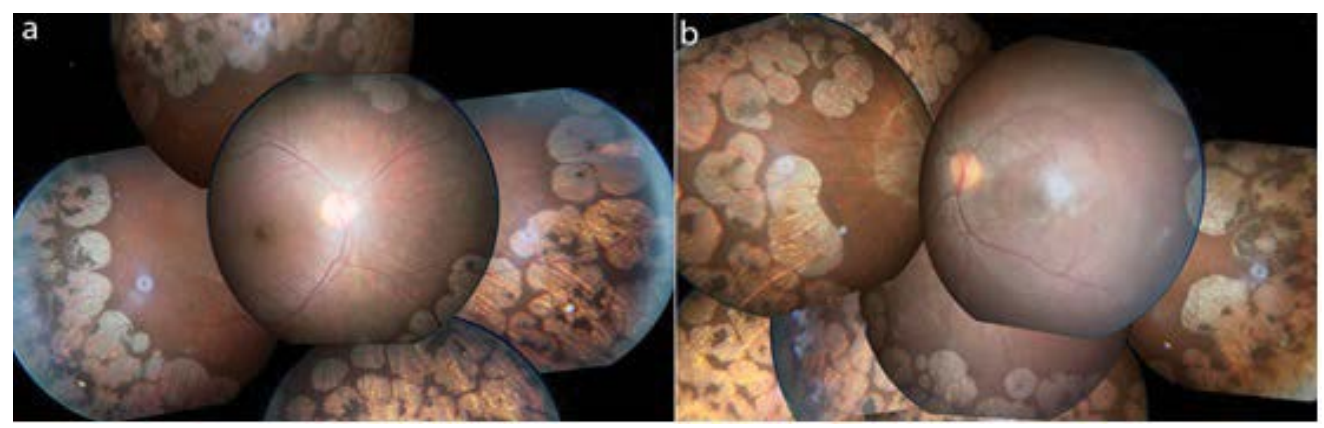

Figure 1: Fundus photography images of the case.

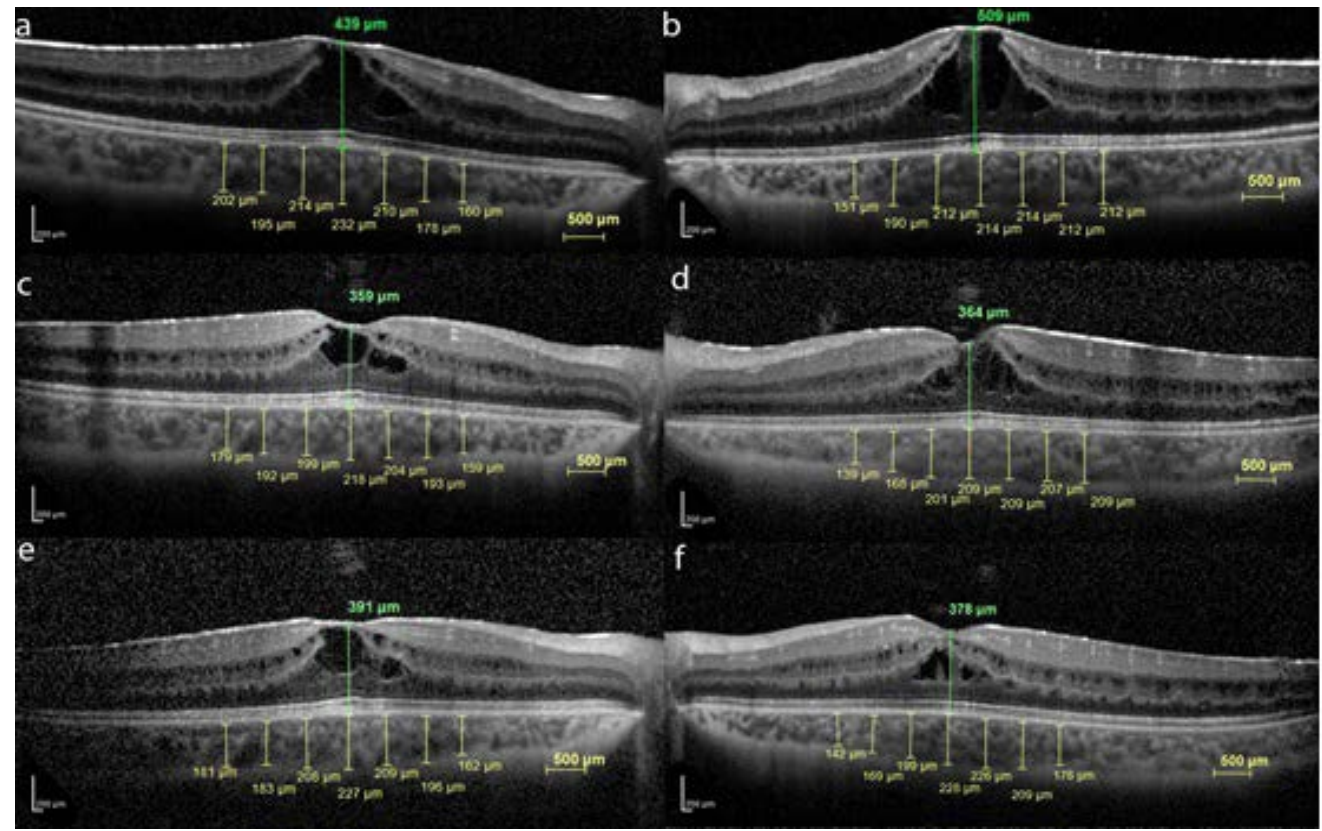

Figure 2: The OCT images of the case.

The macular thickness (MT) and ChT was measured by the SD-OCT (Heidelberg Engineering, Heidelberg, Germany) and it repeated in each monthly visit of the patient. Choroidal thickness was measured perpendicularly from the outer edge of the retinal pigment epithelium to the choroid-sclera boundary at the fovea. Central and at six more points which are located at, respectively, $500 \mu$ nasal to the fovea, $1000 \mu$ nasal to the fovea, $1500 \mu$ to the fovea, $500 \mu$ temporal to the fovea, 1000 $\mu$ temporal to the fovea and $1500 \mu$ temporal to the fovea were noted. The average of these 7 measurements was taken. Choroidal thickness measurements were made by two masked ophthalmologists (FMA and HO). The average of these two measurements was taken.

On the patient's first exam, the MT was measured $439 \mu$ on his RE and $509 \mu$ on his LE. The average ChT were $198.7 \mu$ on the RE, $200.7 \mu$ on the LE (Figure $2 a$ and Figure $2 \mathrm{~b}$ ).

At the $3^{\text {rd }}$ month of the management, even the plasma and urine ornithine level was detected dramatically decreased at $702.05 \mu \mathrm{mol} / \mathrm{L}$ and $934.81 \mu \mathrm{mol} / \mathrm{g} \mathrm{Kre}$ consecutively. His visual acuities in each eye were improved to RE 20/32, LE 20/25. Macular thicknesses were decreased on the RE to $359 \mu$ and the LE to $364 \mu$. And the average ChT were also decreased on the RE to $192 \mu$ and on the LE to $191.7 \mu$ (Figure 2b, Figure $2 \mathrm{c}$ and Figure $2 d)$. On $6^{\text {th }}$ months of the follow-up, BCVA was found decreased, as same level at the baseline (20/40 in the $R E$, and 20/32 in the LE). Macular thicknesses were increased on the RE to $391 \mu$ and the LE to $378 \mu$. And the average ChT were also increased on the RE to $195.1 \mu$ and on the LE to $193 \mu$ (Figure 2e and Figure 2f). Plasma ornithine level was detected at $900.05 \mu \mathrm{mol} / \mathrm{L}$ and the urine ornithine level was detected at $1884.81 \mu \mathrm{mol} / \mathrm{g}$ Kre. The patient was asked if he keep on his diet? He mentioned that he has been taken his B6 tablets, but he abandoned his diet for the last 2 months.

\section{Discussion}

Gyrate atrophy is a rare, autosomal recessive, chorioretinal dystrophy. It has been associated with serum hyperornithinemia due to a deficiency of the vitamin B6-dependent enzyme ornithine ketoacid aminotransferase (OAT), and the human OAT gene has been localized to chromosome 10 and mouse chromosome 7 [5]. Retinitis pigmentosa, Choroideremia and Paving stone degeneration are differential diagnosis of Gyrate Atro- 
phy. Gyrate atrophy can be distinguished in blood and urine enzymes and also the appearance of the fundus.

Typically, clinical appearance of the gyrate atrophy is well-circumscribed atrophic areas in the peripheral retina which include the choroidal and retinal pigment epithelium layer [6]. Cystoid macular edema associated to gyrate atrophy of choroid and retina has been already mentioned in the literature $[4,7]$.

Our case is typical of other gyrate atrophy cases in terms of retinal findings, myopia and serum hyperornithinemia. We have commenced managing the patient with a low-arginine diet and pyridoxine (vitamin B6) supplement and plan to follow his up with periodical plasma ornithine levels, the macular and choroidal thickness measured by SD-OCT. EDI-OCT try to apply but the image was not clear for the measuring and compare. We used the macular grid sections for the comparing the choroidal and macular thickness. Although choroidal thickness is measured by hand, though still surveying the two doctors can give an idea about the choroidal thickness [8].

To be continuing keep on the low-arginine diet is very difficult. The patient could have been followed the diet only a few months. During the patient keep on his diet, the BCVA improved, macular and choroidal thickness were decreased, when the patient gives the diet up, the BCVA decreased, macular and choroidal thickness were increased by the measuring SD-OCT.

In conclusion, this case report highlights the impor- tance of SD-OCT evaluation in the diagnosis of macular and choroidal complication in a patient with gyrate atrophy of the choroid and retina.

\section{References}

1. Francois $\mathrm{J}$ (1982) Metabolic tapetoretinal degenerations. Surv Ophthalmol 26: 293-333.

2. Kaiser-Kupfer MI, Caruso RC, Valle D, Reed GF (2004) Use of an arginine-restricted diet to slow progression of visual loss in patients with gyrate atrophy. Arch Ophthalmol 122: 982-984.

3. Weleber RG, Kennaway NG (1981) Clinical trial of vitamin B6 for gyrate atrophy of the choroid and retina. Ophthalmology 88: 316-324.

4. Vasconcelos-Santos DV, Magalhaes EP, Nehemy MB (2007) Macular edema associated with gyrate atrophy managed with intravitreal triamcinolone: a case report. Arq Bras Oftalmol 70: 858-861.

5. O'Donnell JJ, Vannas-Sulonen K, Shows TB, Cox DR (1988) Gyrate atrophy of the choroid and retina: assignment of the ornithine aminotransferase structural gene to human chromosome 10 and mouse chromosome 7 . Am J Hum Genet 43: 922-928.

6. Moloney TP, O'Hagan S, Lee L (2014) Ultrawide-field fundus photography of the first reported case of gyrate atrophy from Australia. Clin Ophthalmol 8: 1561-1563.

7. Vannas-Sulonen K (1987) Progression of gyrate atrophy of the choroid and retina. A long-term follow-up by fluorescein angiography. Acta ophthalmol 65: 101-109.

8. Sizmaz S, Kucukerdonmez C, Pinarci EY, Karalezli A, Canan $\mathrm{H}$, et al. (2013) The effect of smoking on choroidal thickness measured by optical coherence tomography. $\mathrm{Br} \mathrm{J}$ Ophthalmol 97: 601-604. 